\title{
OBJETIVOS DO DESENVOLVIMENTO SUSTENTÁVEL (ODS-11) E O DIREITO À CIDADE
}

\author{
Sustainable Development Goals (SDG-11) and the Right to the City \\ Objetivos de Desarrollo Sostenible (ODS-11) y Derecho a la Ciudad
}

Érica Nascimento da Silva ${ }^{1}$, João A. Bazzoli ${ }^{* 2}$

${ }^{1}$ Laboratório de Cidades, Bolsista CNPq - Programa Iniciação Cientifica Ensino Médio, Palmas, Brasil.

${ }^{2}$ Laboratório de Cidades, Curso de Direito, Universidade Federal do Tocantins, Palmas, Brasil.

*Correspondência: Laboratório de Cidades, Universidade Federal do Tocantins, Av. NS 15, 109 Norte, Palmas,

Tocantins, Brasil. CEP:77.010-090. e-mail: jbazzoli@uft.edu.br

\section{RESUMO}

Em setembro de 2015, chefes de Estado, de Governo e altos representantes da Organização das Nações Unidas reuniram-se em Nova York e adotaram a Agenda 2030 para o Desenvolvimento Sustentável. Esta Agenda é um plano de ação global que objetiva alcançar os 17 Objetivos de Desenvolvimento Sustentável (ODS) e tem como lema "não deixar ninguém para trás". Para que estes objetivos (ODS) sejam efetivamente alcançados se vislumbra haver necessidade da sua territorialização a partir do contexto global, adaptando-os para às condições nacionais e subnacionais. Este trabalho objetivou analisar qualitativamente as ações locais de aceleração dos Objetivos de Desenvolvimento Sustentável, que foram realizadas em 2019 pelo Programa de Pós-graduação em Desenvolvimento Regional (PPGDR) da Universidade Federal do Tocantins (UFT), decorrentes de atividades extensionistas transcorridas em Palmas-TO. A metodologia qualitativa aplicada ao estudo partiu do acompanhamento das atividades realizadas pelo Programa, da análise de registros acadêmicos e das produções acadêmicas resultantes destas atividades. Concluiu-se com este estudo que para fortalecer e fomentar este tema inter-relacionando-o aos debates acerca do Direito à Cidade, serão necessários a integração destas ações locais acadêmicas com as políticas institucionais (Projetos políticos pedagógicos) e o estabelecimento de parcerias externas, além de maior divulgação do trabalho para a sociedade.

Palavras-chave: Agenda 2030; objetivos de desenvolvimento sustentável; direito à cidade.

\section{ABSTRACT}

In September 2015, heads of state, government and senior representatives of the United Nations met in New York and adopted the 2030 Agenda for Sustainable Development. This Agenda is a global action plan that aims to achieve the 17 Sustainable Development Goals (SDGs) and its motto is "leaving no one behind". In order for these objectives (SDGs) to be effectively achieved, there is a need for their territorialization based on the global context, adapting them to national and subnational conditions. This work aimed to qualitatively analyze the local actions to accelerate the Sustainable Development Goals, which were carried out in 2019 by the Postgraduate Program in Regional Development (PPGDR) of the Federal University of Tocantins (UFT), resulting from extension activities carried out in Palmas- TO. The qualitative methodology applied to the study started from the monitoring of the activities carried out by the Program, the analysis of academic records and the academic productions resulting from these activities. It was concluded with this study that, in order to strengthen and foster this theme, interrelating it to debates on the Right to the City, it will be necessary to integrate these local academic actions with institutional policies (Political Pedagogical Projects) and the establishment of external partnerships, in addition to greater dissemination of the work to society.

Keywords: 2030 agenda; sustainable development goals; right to the city. 


\section{RESUMEN}

En septiembre de 2015, jefes de estado, gobierno y altos representantes de las Naciones Unidas se reunieron en Nueva York y adoptaron la Agenda 2030 para el Desarrollo Sostenible. Esta Agenda es un plan de acción global que tiene como objetivo alcanzar los 17 Objetivos de Desarrollo Sostenible (ODS) y su lema es "no dejar a nadie atrás". Para que estos objetivos (ODS) se logren de manera efectiva, es necesario su territorialización a partir del contexto global, adaptándolos a las condiciones nacionales y subnacionales. Este trabajo tuvo como objetivo analizar cualitativamente las acciones locales para acelerar los Objetivos de Desarrollo Sostenible, que fueron realizadas en 2019 por el Programa de Posgrado en Desarrollo Regional (PPGDR) de la Universidad Federal de Tocantins (UFT), resultado de las actividades de extensión realizadas en Palmas. - PARA. La metodología cualitativa aplicada al estudio partió del seguimiento de las actividades realizadas por el Programa, el análisis de los expedientes académicos y las producciones académicas resultantes de estas actividades. Con este estudio se concluyó que, para fortalecer y fomentar este tema, interrelacionándolo con los debates sobre el Derecho a la Ciudad, será necesario integrar estas acciones académicas locales con las políticas institucionales (Proyectos Político Pedagógicos) y el establecimiento de alianzas externas, además de una mayor difusión del trabajo a la sociedad.

Descriptores: Agenda 2030; Metas de desarrollo sostenible; Derecho a la Ciudad.

\section{INTRODUÇÃO}

Pontua-se que em 2015, na Assembleia Geral das Nações Unidas (ONU), aprovou-se o documento denominado "Transformando nosso mundo: A Agenda 2030 para o Desenvolvimento Sustentável". (ONU, 2016)

Esse novo acordo sucedeu a pauta dos Objetivos de Desenvolvimento do Milênio (ODM), onde os países membros da ONU deveriam atingir 8 Objetivos Globais de Desenvolvimento até o ano de 2015.

Verificou-se então, que os ODM foram substituídos pela nova Agenda 2030, dos Objetivos de Desenvolvimento Sustentável (ODS).

O fato é que os Objetivos de Desenvolvimento Sustentáveis (ODS) são globais por natureza e universalmente aplicáveis, ou seja, suas ações de implantação devem ser adaptadas às realidades nacionais e subnacionais. Neste sentido a ONU passou a incentivar os países membros a adotar este acordo, isto a partir das metas globais que compõem os 17 Objetivos de Desenvolvimento Sustentáveis (ODS), adaptando-os às suas metas nacionais e, com as devidas adequações, aos contextos subnacionais. (SILVA, 2018).
Trazendo ao conhecimento um dos lemas mais memoráveis da Organização das Nações Unidas (ONU), que diz que devemos "Pensar globalmente e agir localmente", buscou-se com este estudo entender como se apropriar da Agenda 2030, analisando mecanismos locais que pudesse fomentar a efetivação da cidadania global, no sentido de assegurar o Direito à Cidade com a promoção de ações acadêmicas propositivas. (ONU, 2016).

Neste sentido, temos de pontuar que o Direito à Cidade é entendido pelo Estatuto da Cidade como aquele que proporciona o acesso à terra urbana, à moradia, ao saneamento ambiental, à infraestrutura urbana, ao transporte e aos serviços públicos, ao trabalho e ao lazer, para a presente e futuras gerações (Lei $\mathrm{n}^{\mathrm{o}}$ 10.257, de 10 de julho de 2001, Art. $2^{\circ}$, Inciso I), alinha-se integralmente à Agenda 2030, ODS 11 Cidades e comunidades sustentáveis.

Nesta mesma linha de análise agregamos ao debate a "Nova Agenda Urbana" (NAU) documento adotado pela ONU-Habitat no ano de 2016, na Conferência das Nações Unidas sobre Habitação e Desenvolvimento Urbano Sustentável, conhecida como Habitat III. que serve como guia de orientação para uma urbanização saudável para os próximos 20 
anos e representa uma visão compartilhada para um futuro melhor e mais sustentável.

Este documento compartilha a visão de cidades para todos e todas, trata da igualdade, promove a inclusão, assegura a habitação, mas essencialmente relaciona esta visão ao "direito à cidade", termo transcrito no item 11 da Agenda (NAU). (GOMES et al, 2019).

Diante deste contexto, este trabalho objetivou analisar qualitativamente as ações locais de aceleração dos Objetivos de Desenvolvimento Sustentável, que foram realizadas em 2019 pelo Programa de Pósgraduação em Desenvolvimento Regional (PPGDR) da Universidade Federal do Tocantins (UFT), decorrentes de atividades extensionistas transcorridas em PalmasTO.

Para realizar a análise proposta foi necessário inicialmente compreender as diretrizes aplicáveis aos ODS a partir da realidade da Cidade de Palmas.

Observou-se que as atividades analisadas pelo estudo tinham a finalidade de propagar a Agenda 2030, tanto para a comunidade acadêmica como para a sociedade local, através de ações concretas de aceleração de implantação dos ODS. E, isto com a finalidade de fortalecer e fomentar, para além de atividades relacionadas a prática dos ODS, mas a atuação no campo da Educação para o Desenvolvimento Sustentável (EDS), bem como, pretendeu-se ampliar os debates acerca da Nova Agenda Urbana (NAU) e o ODS 11, no sentido de ampliar o debate local, também sobre o Direito à Cidade.

Nota-se que, em havendo uma urbanização planejada e exitosa, vários ODS serão alcançados.

\section{MATERIAIS E MÉTODOS}

A metodologia qualitativa aplicada ao estudo partiu do acompanhamento das atividades realizadas, da análise de registros acadêmicos e das produções resultantes destas atividades. Buscou-se também, o aprofundamento temático via documental e a revisão de literatura específica, com abordagem nos Objetivos de Desenvolvimento Sustentável (ODS), seguida de análise a partir da realidade de Palmas.

Feito isto foi possível entender o mecanismo de territorialização da Agenda 2030 e de como é possível promover a Educação para o Desenvolvimento Sustentável (EDS), como instrumento de divulgação dos ODS na comunidade escolar palmense.

Segundo a UNESCO (Organização das Nações Unidas para Educação, Ciência e Cultura) a Educação para o Desenvolvimento Sustentável (EDS) contribui para mudar a forma como as pessoas pensam e agem para alcançarmos um futuro sustentável. Assim, conseguimos entender a importância de trabalharmos acerca desse tema nas escolas de Palmas.

Neste contexto de aprofundamento temático, realizou-se um levantamento em pontos do Plano Diretor Participativo do Município de Palmas-TO à luz do ODS 11 (Cidades e Comunidades Sustentáveis) e da Nova Agenda Urbana (NAU), para identificar a integração da legislação municipal com a ações de efetivação do Direito à Cidade (BAZZOLI, 2019).

Importante salientar que para a realização deste estudo, para além da pesquisa bibliográfica através de materiais como documentos, artigos e livros, ocorreu a participação efetiva dos pesquisadores nas ações desenvolvidas e estudadas.

Outro ponto relevante foi que para o aprimoramento do estudo os autores participaram durante os estudos do Curso "Integrando a Agenda 2030 para os Objetivos de Desenvolvimento 
Sustentável" promovido pelo PNUD (Programa das Nações Unidas para o Desenvolvimento), onde foi possível o aprofundamento temático teórico e prático sobre a Agenda 2030 e sua territorialização.

Assim sendo, com a integração dos pesquisadores com o objeto foi possível, para além de proceder análise, mas de apontar concretamente os pontos convergentes e conflitantes entre os instrumentos e os mecanismos de aceleração para a implementação dos ODS em sede local.

Isto, com a finalidade de contribuir para que a sociedade efetivamente tivesse um maior aprofundamento dos conhecimentos sobre os ODS para propagá-los e fortalecê-los, a partir de formulação de políticas públicas integradoras, que integrem efetivamente o poder transformador da urbanização.

\section{RESULTADOS E DISCUSSÃO}

Como resultados foram acompanhadas ações fundamentais promovidas para a disseminação temática no sentido educativo e propositivo.

Importante salientar também a intenção integrativa na realização deste trabalho, como o de buscar o nivelamento e o aprofundamento teórico pelos pesquisadores, com a participação nas ações e nos cursos realizados sobre o tema.

E, neste contexto geral da análise que foi realizada pelo estudo, destaca-se para mostrar neste artigo ações pontuais, porém relevantes, pelo seu caráter multiplicador, tanto quantitativo como qualitativo.

Desta maneira iniciamos pelo Fórum Municipal, que aconteceu no dia 12 de setembro de 2019, na sede da ATM (Associação Tocantinense de Municípios). Neste evento realizado a partir de seminário presencial que tratou da "Articulação da Implementação do Centro de Desenvolvimento
Regional Metropolitana Palmas na perspectiva da Agenda 2030", mostrado na figura 1, buscou-se contribuir para a abertura de espaço para debates referentes ao Desenvolvimento Regional à luz da Agenda 2030, bem como contribuir para um aprofundamento teórico no que diz respeito às cidades e ao ODS 11.

Figura 1. Reunião com lideranças regionais.

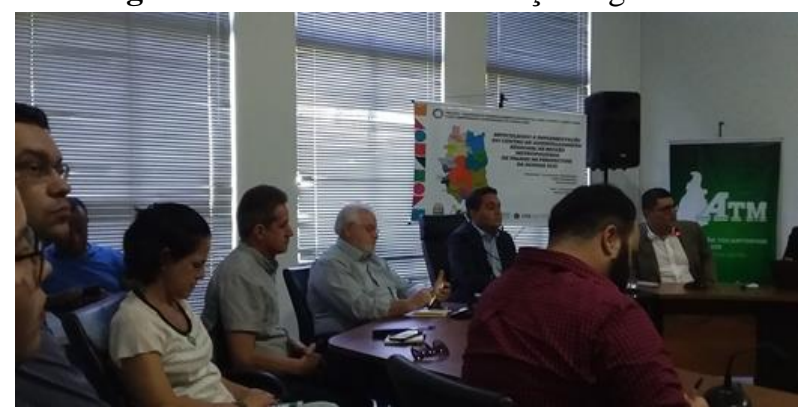

Fonte: arquivo pessoal dos autores, 2019.

Importante salientar os reflexos da ação em razão de ser publicada no "Jornal Joseense” de São José dos Campos - SP, pelo site "T1 Notícias”, o site “Conexão Tocantins", estas mídias da cidade de Palmas. Pontua-se que outras mídias publicaram noticia a respeito do evento, segundo (Bazzoli et al, 2019, p. 74) as mídias obtiveram 1917 acessos.

Esta atividade resultou na publicação do artigo “A articulação e implementação do centro de desenvolvimento regional na região metropolitana de Palmas: um relato de experiência”, em Revista de Extensão especializada. (BAZZOLI et al, 2019).

$\mathrm{Na}$ sequência das atividades, analisou-se o evento "Circuito Urbano 2019", que aconteceu no dia 25 de outubro de 2019, onde foram apresentados trabalhos acadêmicos do Programa de Pós-graduação em Desenvolvimento Regional (PPGDR-UFT), relacionados a ações de aceleração de implementações dos ODS, conforme demonstrado na figura 2. 
Figura 2. Palestra no evento Circuito Palmas 2019.

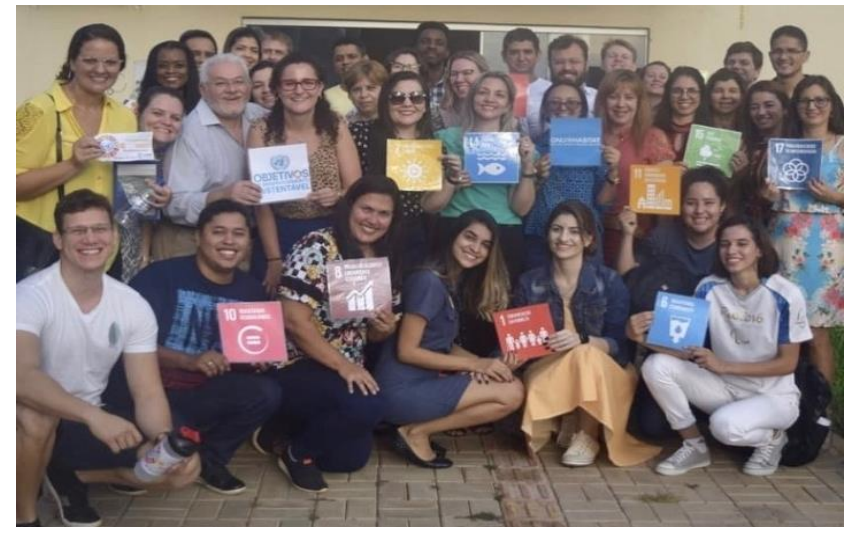

Fonte: arquivo pessoal dos autores, 2019.

O evento do Circuito Urbano 2019 possibilitou avaliar os impactos em Palmas, a partir da análise das ações do Projeto "Objetivos de Desenvolvimento Sustentável (ODS): atores e ações locais para compreensão e apropriação da Agenda 2030", desenvolvido em parceria com a sociedade em geral.

Este evento também mostrou os projetos/ações realizadas pelos acadêmicos do mencionado Programa, mostrado na figura 3 , sobre os ODS e despertou a importância e a compreensão da aplicabilidade dos ODS na prática, na cidade de Palmas.

Figura 3. Apresentação de trabalhos.

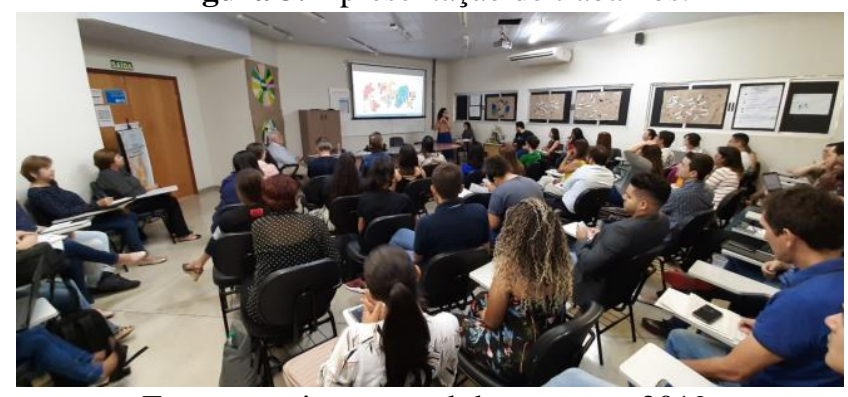

Fonte: arquivo pessoal dos autores, 2019.

Pretendia-se com esta atividade disseminar informações sobre os ODS por meio de ações práticas realizadas na comunidade.

Entre os trabalhos apresentados se destacou: i. "Objetivos de desenvolvimento sustentável: uma análise na unidade prisional feminina de Palmas-TO" esta atividade realizou formação de 15 reeducandas; ii.
Energia solar: um estudo em Palmas, discutiu esta política pública adotada pela Prefeitura para ampliar o campo de oferta de energia renovável; iii. Desafios da aplicação dos ODS em uma escola, através de jogos lúdicos propôs a discussão dos ODS em escola da Região Norte de Palmas-TO; iv. Projeto Eco Eletro Gestão do lixo eletrônico para o desenvolvimento sustentável, recolheu lixo eletrônico e por meio de palestras apresentou a Agenda 2030 e por fim; v. A experiência do Projeto Vozes: território integrado à Agenda 2030, realizou a formação de assessores populares em ODS no setor Taquari em Palmas-TO.

Esta última atividade possibilitou a formação de 50 moradores deste bairro periférico da cidade. Para além disso, o Projeto foi adotado para ser replicado e continua propiciando a formação de populares no tema.

Importante salientar, no mesmo propósito de divulgar os ODS foram realizadas Lives (apresentações pela internet) e entre elas no dia 20/08/2020, transmitida pelo canal do YouTube "Cidade Vivida", foi apresentado o trabalho “Agenda 2030. Que papo é esse? A atividade buscou alcançar jovens estudantes do Ensino Médio da rede estadual tocantinense de educação e contou com a audiência de mais de 385 acessos, demonstrado na figura 4 . Por pretender trazer de forma clara e adaptada à uma linguagem acessível, o que é a Agenda 2030 e alguns exemplos de trabalhos realizados em Palmas que promovem o alcance dos ODS, a proposta conseguiu atingir o objetivo. Esse evento foi importante pois foi possível expor a Agenda 2030 para os jovens de uma forma clara e simplificada, sendo assim, possível promover efetivamente a Educação para o Desenvolvimento Sustentável (EDS) de uma forma acessível e lúdica.

Figura 4. Agenda 2030. Que papo é esse?" 


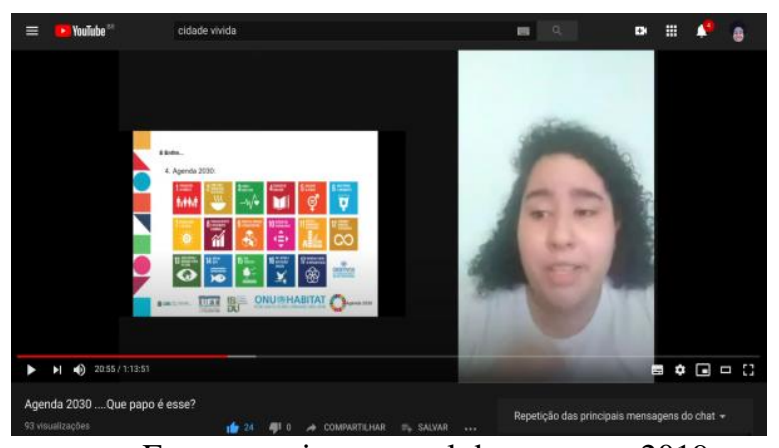

Fonte: arquivo pessoal dos autores, 2019.

Pontua-se pela importância do fato que as atividades realizadas, embora estivessem ligadas ao conteúdo das disciplinas especificas: Seminários Interdisciplinares de Desenvolvimento Regional I e II, foram interligas ao Projeto de extensão "Objetivos de Desenvolvimento Sustentável (ODS): atores e ações locais para compreensão e apropriação da Agenda 2030 " e receberam apoio institucional do Programa das Nações Unidas para os Assentamos Humanos (ONUHabitat), do Instituto Brasileiro de Direito Urbanístico (IBDU) e o Conselho de Arquitetura e Urbanismo do Tocantins (CAU/TO), por meio da Comissão de Planejamento Urbano e Meio Ambiente (CEPUA).

\section{CONCLUSÃO}

Conclui-se com este trabalho que atender aos Objetivos de Desenvolvimento Sustentáveis é uma questão de sobrevivência e que é necessário possibilitar a formação de cidadãos globais.

Também, descobriu-se a necessidade da promoção de debates constantes deste tema para fazer valer a função do Direito à cidade e da vida urbana sustentável. Percebeu-se ainda, a importância de uma maior promoção dos ODS para a população da cidade de Palmas, em razão de ser uma cidade jovem e com grande potencial para ser referência de sustentabilidade no âmbito de proteção à vida terrestre e urbana.

Observou-se o desconhecimento local sobre o tema e poucas iniciativas concretas voltadas para suprir esta necessidade, considerando a importância global do assunto.

Salienta-se que estas ações realizadas no ano de 2019 resultaram na criação de um site específico pela Universidade Federal do Tocantins (UFT) para depositar os acervos das ações realizadas de aceleração dos ODS, encontrado no endereço eletrônico: https://sites.google.com/view/odsuft.

Foi criado um Canal de Youtube para transmitir eventos e depositar vídeos com as ações locais, encontrado no endereço eletrônico: https://www.youtube.com/channel/UCJwFVHMrthze Wd0sMkjok2g.

Observa-se várias iniciativas institucionais tratando dos ODS, como a inclusão do tema na Política de Extensão e no Plano de Desenvolvimento Institucional (PDI), bem como, a inserção definitiva do tema no ensino, pesquisa e extensão do Programa de Pós-graduação em Desenvolvimento Regional (PPGDR) e a criação no Curso de Direito da disciplina “Agenda 2030 para o Desenvolvimento Sustentável".

Logo, denota-se também a importância da propagação dos Objetivos de Desenvolvimento Sustentável no ambiente escolar, para que assim os jovens e as crianças compreendam a importância de ações e estilos de vida sustentáveis.

Destarte, concluímos também, que o estudo dos Objetivos de Desenvolvimento Sustentável (ODS) é fundamental para a integração do poder de formulação de políticas públicas para alcançarmos os objetivos e metas da Agenda 2030.

\section{AGRADECIMENTOS}

Este trabalho foi REALIZADO com o apoio do Conselho Nacional de Desenvolvimento Científico e Tecnológico $-\mathrm{CNPq}-$ Brasil e foi PREMIADO no 
XVI Seminário de Iniciação Cientifica (SIC) da Universidade Federal do Tocantins (UFT).

Todos os autores declararam não haver qualquer potencial conflito de interesses referente a este artigo.

\section{REFERÊNCIAS}

BAZZOLI, João A. Palmas em foco: contradições de uma cidade planejada. Palmas: EDUFT, 2019.

BAZZOLI, J., LIMA, F.S, BORGES, H.R.P.S.S, ALVES, J.J.D., RODRIGUES, S.G., RODRIGUES, W. A articulação e implementação do centro de desenvolvimento regional na região metropolitana de Palmas: um relato de experiência. Revista Extensão, v.3, n. 2. nov. 2019. pp. 71-80.

GOMES, Déborha Souza Alves; SILVA, Érica Nascimento da; BAZZOLI, João Aparecido. Regularização Fundiária em Palmas-TO: Análise à Luz da Agenda 2030. In: Seminário Internacional O Direito
Como Liberdade: 30 anos de O Direito Achado na Rua, 2019, Brasília. Anais [...]. Brasília: Universidade de Brasília, 2020. p.1-16. Disponível em: https://direitoachadonarua.files.wordpress.com/2020/0 7/gt-7-gomes-bazolli-e-silva.pdf Acesso em: Set.2020

NAÇÕES UNIDAS NO BRASIL (ONU). 17 objetivos para transformar nosso mundo. ONU, 2016. Disponível em: https://nacoesunidas.org/pos2015/agenda2030/. Acesso em dez. 2018.

NAÇÕES UNIDAS NO BRASIL (ONU). Roteiro para a Localização dos Objetivos de Desenvolvimento Sustentável: Implementação e Acompanhamento no nível subnacional. ONU, 2016. Disponível em: https://nacoesunidas.org/wpcontent/uploads/2017/06/Roteiro-para-a-Localizacaodos-ODS.pdf . Acesso em: nov. 2019.

SILVA, Enid Rocha Andrade da (Coordenadora). Agenda 2030: ODS-Metas nacionais dos objetivos de desenvolvimento sustentável. IPEA. 2018. Disponível em: http://repositorio.ipea.gov.br/handle/11058/8855. Acesso em nov.2019. . 